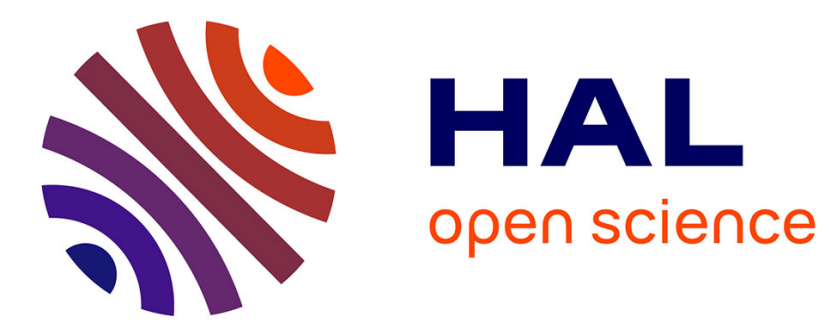

\title{
Etude de la dispersion normale des caractéristiques auditives
}

\author{
G. Canévet, A. Marchioni
}

\section{To cite this version:}

G. Canévet, A. Marchioni. Etude de la dispersion normale des caractéristiques auditives. Journal de Physique IV Proceedings, 1994, 04 (C5), pp.C5-357-C5-366. 10.1051/.jp4:1994574 . jpa-00253069

\section{HAL Id: jpa-00253069 https://hal.science/jpa-00253069}

Submitted on 1 Jan 1994

HAL is a multi-disciplinary open access archive for the deposit and dissemination of scientific research documents, whether they are published or not. The documents may come from teaching and research institutions in France or abroad, or from public or private research centers.
L'archive ouverte pluridisciplinaire HAL, est destinée au dépôt et à la diffusion de documents scientifiques de niveau recherche, publiés ou non, émanant des établissements d'enseignement et de recherche français ou étrangers, des laboratoires publics ou privés. 


\title{
Etude de la dispersion normale des caractéristiques auditives
}

\author{
G. CANÉVET et A. MARCHIONI
}

Laboratoire de Mécanique et d'Acoustique, CNRS, 13402 Marseille cedex 20, France

\begin{abstract}
Auditory performances were measured on two groups of normally-hearing listeners, for a total of 107 persons, whose ages ranged from 14 to 50 with a majority between 20 and 25 . Aside from the classical audiogram, the variable tested were absolute and masked detection thresholds for one part, and discrimination thresholds for the rest. The main goal of this test battery was to get an estimate of the normal distribution of auditory capabilities for intensity, frequency, time and space variables; but we also measured binaural fusion, stream segregation, timbre discrimination, and selectivity in general. The objective behind this investigation is to build a simplified model for auditory perception, based on a multidimensional representation. In this model, the main features of auditory perception could be represented by independant vectors, and each individual by his components relative to these vectors. A statistical analysis of our data show that it is not perfectly clear yet what the independant factors are. Nevertheless, a "psychoacoustic profile" can be defined, for each individual or for groups of individuals, based on a limited set of variables, that might prove to be a useful tool for some professional applications.
\end{abstract}

\section{INTRODUCTION.}

La perception de l'environnement sonore varie beaucoup d'un individu à l'autre. Cela tient à la diversité des significations évoquées par les signaux acoustiques. Mais cela vient aussi de ce que les capacités auditives individuelles sont inégalement réparties; elles le sont entre les individus d'abord, mais aussi pour un même individu d'une fonction auditive à l'autre : un excellent seuil de détection absolu ne s'accompagne pas forcément d'une bonne capacité de discrimination de hauteur par exemple. On peut tout de même faire l'hypothèse que la perception auditive se construit à partir d'un nombre limité de dimensions fondamentales (détection, acuité fréquentielle, temporelle etc...), que l'on pourrait appeler vecteurs de base psychoacoustiques, par analogie avec l'algèbre linéaire. Toute personne pourrait alors être caractérisée par une série de composantes, chacune se rapportant à un des vecteurs de la base, donc à une dimension donnée de la perception auditive.

Ce type de modélisation de la perception auditive correspond à une démarche déjà exploitée dans d'autres domaines. On connaît par exemple les profils psychologiques de Carton [2], qui se décrivent simplement par combinaison de quatre types fondamentaux : bilieux, sanguin, nerveux et lymphatique. Plus proche de nous, les travaux de Kuder [5] sur les inclinations professionnelles ont permis la définition d'une série de dix facteurs de base (numérique, social, artistique, persuasif etc...) considérés comme représentatifs des composantes principales du choix d'un individu. Les tests mis au point pour mesurer le degré de développement individuel de ces facteurs sont couramment utilisés pour évaluer les "préférences professionnelles" des sujets testés. Appliquée à l'audition, une étude équivalente à celle de Kuder permettrait peut-être d'aboutir à la définition d'un profil psychoacoustique individuel. Il faudrait pour cela définir les fonctions auditives de base, puis établir une batterie de tests qui en permettent la mesure. 
Un certain nombre de chercheurs se sont intéressés à ce problème, et nous reportons le lecteur à une autre publication [1] pour une analyse des acquis de leurs travaux. Cependant la question reste tout à fait ouverte, car il n'a pas encore été possible de déterminer avec certitude les facteurs auditifs de base. Nous avons donc entrepris une étude dans ce but, dont nous présentons ici quelques résultats. Cette étude a consisté à dresser un bilan audiométrique de sujets sains, au moyen d'une batterie de tests choisis de manière à représenter au mieux l'ensemble des dimensions de la perception auditive. Cest tests mesurent essentiellement des seuils de détection, absolus ou masqués, et des seuils de discrimination. Les résultats semblent confirmer que la représentation en facteurs est possible. Une analyse discriminante montre que l'on peut aussi définir des catégories auditives, c'est-à-dire des regroupements de sujets ayant des profils voisins. Mais la dispersion des données est continue, et il n'y a pas à proprement parler d'amas indépendants (clusters) dans les échantillons que nous avons testés.

\section{DESCRIPTION DES TESTS, MÉTHODE, SIGNAUX.}

Cette étude sur les différences interindividuelles dans la détection et la discrimination auditives s'est déroulée en deux temps. Tout d'abord nous avons procédé à une première série de quinze tests, sur un ensemble de 51 personnes. Les résultats de cette première partie sont présentés dans la note déjà citée [1]. Une seconde série de tests a ensuite été pratiquée, celle que nous présentons ici, sur un nouvel échantillon de 56 personnes. Ces tests, qui ont permis la mesure de 19 variables (voir les tableaux I et II), sont les mêmes tests que ceux de la première, à quelques modifications de détail près pour deux d'entre eux. Nous en rappelons brièvement la description.

\subsection{Description des tests}

1) Discrimination de durées : deux signaux consécutifs sont présentés, l'un d'une durée fixe de $100 \mathrm{~ms}$, l'autre $100 \mathrm{~ms}+$ DT. L'ordre est choisi au hasard, le sujet doit dire lequel est le plus long. La valeur de DT est fixée à $100 \mathrm{~ms}$ au départ.

2) Discrimination de durées de pauses : le signal est constitué de deux impulsions de bruit séparées par une pause. On présente deux fois un tel signal, avec une pause de100 ms pour l'un, et 100+DT pour l'autre; au maximum DT=100 ms. Il s'agit de reconnaître la pause la plus longue.

3) Discrimination de rythmes (test adapté de Johnson et Watson, [4]) : on présente au sujet, trois fois consécutives, deux impulsions de bruit. L'ensemble fait donc six impulsions, intervenant à une certaine cadence : la durée d'une bouffée est de $20 \mathrm{~ms}$, l'intervalle de silence entre les deux bouffées d'une paire est de $40 \mathrm{~ms}$, et l'intervalle de silence entre deux paires consécutives est de $80 \mathrm{~ms}$. Une telle séquence de six impulsions constitue le signal de référence, et donc le rythme de référence.

Une seconde après avoir présenté la séquence de référence, on présente au sujet deux séquences supplémentaires, séparées par un silence de $500 \mathrm{~ms}$ : l'une a exactement le même rythme que la référence, l'autre a un rythme légèrement différent. L'intervalle entre deux bouffées de chaque paire est porté à 40+DT $\mathrm{ms}$, et l'intervalle entre paires réduit en conséquence à 80 -DT ms. Le sujet doit indiquer la séquence qui reproduit le rythme de référence.

4) Fusion interaurale : deux sons de fréquences différentes, F1 et F2 sont présentés simultanément, un sur chaque oreille, la fréquence moyenne Fc est de $1 \mathrm{kHz}$. Le sujet doit dire de quel côté est le son le plus aigu.

5) Discrimination fréquentielle: Le sujet doit dire lequel de deux sons, $\mathrm{F}$ et $\mathrm{F}+\mathrm{dF}$, qui lui sont présentés est le plus aigu. On commence avec un écart $\mathrm{dF}=\mathrm{F} / 20$. De plus, on introduit à chaque son présenté une petite variation aléatoire de niveau, comprise dans les limites de $\pm 2,5 \mathrm{~dB}$, autour de la valeur nominale.

6) Discrimination de séquences : Le signal est constitué de 4 sons successifs, constituant une séquence du type Do-Mi-La-Do. Le premier et le dernier son durent $100 \mathrm{~ms}$, et la durée DT des deux sons intermédiaires est variable. Au départ du test, $\mathrm{DT}=50 \mathrm{~ms}$. Le test consiste à présenter d'abord une séquence de référence, comme celle qui vient d'être décrite. Puis, une seconde plus tard, on présente deux autres séquences, séparées par un silence de $500 \mathrm{~ms}$. L'une de ces deux séquences est identique à la référence. Dans l'autre, les sons intermédiaires sont inversés; autrement dit, l'une des séquences est encore Do-Mi-La-Do, mais l'autre Do-La-Mi-Do. Le sujet doit indiquer laquelle de ces deux séquences reproduit la référence. Ce test est aussi adapté de Johnson et Watson [4]. 
7) Masquage informationnel : ce test est comparable au précédent [8], excepté que la séquence comprend 10 sons, de $45 \mathrm{~ms}$ chacun, accolés sans intervalles de silence, à dix fréquences différentes. On mesure le seuil de détection du huitième de ces sons, pour un niveau fixe des neuf autres.

8) Détection dans le bruit : test classique de détection, où l'on présente deux bouffées successives de bruit, l'une contenant un son pur surajouté. La mesure porte sur le niveau minimum détectable du son pur.

9) Mesure de MLD, ou démasquage (masking level difference). Rappelons que le MLD est la différence des seuils de détection d'un signal dans le bruit obtenus dans deux conditions d'écoute: l'une où le signal est en phase sur les deux oreilles, l'autre où il est en opposition. Le bruit est en phase dans les deux cas. La mesure se fait comme pour le test précédent.

10) Sélectivité temporelle, antérieure et postérieure, mesurée par un test de masquage selon une procédure inspirée de Fastl [3]. Le masque est un bruit à large spectre et on mesure le seuil de détection d'un son bref (3 ms) à $8 \mathrm{kHz}$ présenté avec un certain décalage temporel, à savoir juste avant, pendant ou exactement au début de la bouffée de bruit.

11) Discrimination de spectres : reprenant une expérience de Santon [6] sur la coloration d'un bruit par addition de sa copie retardée, ce test mesure la capacité à détecter un changement de timbre. Un bruit est d'abord présenté seul, puis deux intervalles sont présentés : l'un d'eux est la simple répétition de la référence, l'autre est composé du bruit de référence et du même bruit retardé de $10 \mathrm{~ms}$, et atténué. On cherche le niveau minimum de cette composante retardée au seuil de détection.

12) Discrimination de niveaux : Construit comme les tests de discrimination de fréquence ou de durée, ce test mesure la sensibilité différentielle des sujets pour le niveau acoustique, c'est-à-dire la différence minimale perceptible $\mathrm{dL}$ entre signaux de niveaux $\mathrm{L}$ et $\mathrm{L}+\mathrm{d} \mathrm{L}$

13) Discrimination de flux, mesurée par le CTS de Trenque et König [7] : il s'agit de mesurer le seuil de masquage d'un signal de la zone du médium par un signal plus grave: les deux signaux sont des glissandos continus, à variation linéaire de fréquence, le son test de 2 à $4 \mathrm{kHz}$, le masquant de 1 à $2 \mathrm{kHz}$. Ils sont simultanés, durent $200 \mathrm{~ms}$, leur établissement dure $15 \mathrm{~ms}$ et leur extinction $5 \mathrm{~ms}$.

14) Seuil de latéralisation d'écho : comme le MLD, ce test est une forme de mesure des capacités de perception spatiale. On fait entendre au sujet une bouffée de bruit de $10 \mathrm{~ms}$, simultanément sur chaque oreille. Il perçoit donc ce bruit au centre de la tête. Après un silence de $500 \mathrm{~ms}$, on lui fait entendre successivement la même bouffée, au même niveau que la première, puis $22 \mathrm{~ms}$ plus tard son "écho" c'est-àdire une copie atténuée de cette bouffée, avec en plus un retard interaural de $1 \mathrm{~ms}$ qui provoque la sensation d'entendre l'écho du côté de l'oreille stimulée en avance. La mesure porte sur le niveau minimal de l'écho pour lequel le sujet peut identifier ce côté, qui est choisi au hasard à chaque présentation.

\subsection{Méthode}

La majorité des tests a été effectuée à l'aide d'une méthode à choix forcé avec feedback, généralement à deux intervalles sauf pour les tests $\mathrm{n}^{\circ} 3,6,7$ et 11 où il y en avait trois. Seul le CTS de Trenque et König $\left(\mathrm{n}^{\circ} 13\right)$ utilisait une procédure de "poursuite" de Békésy, conformément à la définition du test par ses auteurs. L'approche des seuils se faisait selon une procédure adaptative, avec augmentation de la difficulté après deux réponses correctes consécutives, et abaissement après chaque réponse incorrecte. Le test était suspendu après six inversions de la variable; la moyenne des valeurs aux quatre dernières inversions servant au calcul d'un seuil partiel. Puis la mesure reprenait pour une nouvelle estimation du seuil recherché. Il y avait au total trois estimations de la sorte, dont la moyenne, arithmétique ou géométrique suivant le cas (voir §3.1), était retenue comme valeur du seuil.

Lorsque les mesures portaient sur le niveau, les pas de variations étaient de $5 \mathrm{~dB}$ jusqu'à la seconde inversion, et de $2 \mathrm{~dB}$ ensuite. Lorsque les mesures portaient sur des rapports (de durées, de fréquences), les variations se faisaient par multiplication, ou division suivant le cas, par un coefficient 2 jusqu'à la seconde inversion, et 1,5 par la suite.

Les tests étaient gérés par ordinateur. Ils se déroulaient dans une cabine audiométrique, équipée d'un terminal de réponse pour le sujet. La présentation des signaux, à l'aide d'écouteurs AKG K340, était dichotique pour les tests $n^{\circ} 4,9$ et 14 , et diotique pour les autres. 


\subsection{SIGNAUX}

Certains tests portaient uniquement sur des sons purs ou des bruits (de largeur spectrale $\Delta F$ ), d'autres combinaient les deux. Les caractéristiques des signaux sont résumées dans le tableau I. Leurs durées d'établissement et d'extinction étaient de $1 \mathrm{~ms}$ pour les tests 10 et 14 , et de $5 \mathrm{~ms}$ pour les autres.

Tableau I. Caractéristiques des signaux utilisés pour les tests.

\begin{tabular}{|c|c|c|}
\hline$N^{\circ}$ du Test, Variables & Signaux & Bruits \\
\hline 1) DTM & & $\Delta \mathrm{F}=100-15000 \mathrm{~Hz}, 65 \mathrm{~dB} \mathrm{SPL},(100 / 100+\mathrm{DT}) \mathrm{ms}$ \\
\hline 2) DGM & & $\Delta \mathrm{F}=100-15000 \mathrm{~Hz}, 70 \mathrm{~dB} \mathrm{SPL},(200 / 100+\mathrm{DT} / 200) \mathrm{ms}$ \\
\hline 3) RYM & & $\begin{array}{l}\Delta \mathrm{F}=100-15000 \mathrm{~Hz}, 65 \mathrm{~dB} \text { SPL, Réf : }(20 / 40 / 20 / 80) \mathrm{ms}, \\
\text { Test : }(20 / 40+\mathrm{DT} / 20 / 80-\mathrm{DT}) \mathrm{ms}\end{array}$ \\
\hline 4) FUM & $\begin{array}{l}F 1{ }^{*} \mathrm{~F} 2=\mathrm{F}_{\mathrm{c}}{ }^{2}, \mathrm{FC}=1000 \mathrm{~Hz}, 350 \mathrm{~ms}, 70 \mathrm{~dB} \\
\mathrm{SPL}\end{array}$ & \\
\hline 5) $\mathrm{DF}(1-4) \mathrm{M}$ & 1 ou $4 \mathrm{kHz}, 350 \mathrm{~ms}, 70 \mathrm{~dB}$ SPL & \\
\hline 6) TSM & $\begin{array}{l}4 \text { sons : } 625,710,550,625(\mathrm{~Hz}) \\
100 / 10+\mathrm{DT} / 10+\mathrm{DT} / 100 \mathrm{~ms}, 70 \mathrm{~dB} \text { SPL }\end{array}$ & \\
\hline 7) TTM & $\begin{array}{l}10 \text { sons : } 1324,1800,768,2339,1863, \\
620,1184,948,1633,2620(\mathrm{~Hz}), 45 \mathrm{~ms} \\
70 \text { dB SPL }\end{array}$ & \\
\hline 8) $\mathrm{BR}(1-1,8) \mathrm{M}$ & 1 et $1,8 \mathrm{kHz}, 350 \mathrm{~ms}$ & $\Delta \mathrm{F}=200-1300 \mathrm{~Hz}, 700 \mathrm{~ms}, \Delta \mathrm{T}(\mathrm{B} / \mathrm{S})=250 \mathrm{~ms}, 65 \mathrm{~dB} \mathrm{SPL}$ \\
\hline 9) $\mathrm{MLD}(0-1) \mathrm{M}$ & $500 \mathrm{~Hz}, 200 \mathrm{~ms}$ & $\begin{array}{l}\Delta \mathrm{F}=300-700 \mathrm{~Hz}, 500 \mathrm{~ms}, \Delta \mathrm{T}(\mathrm{B} / \mathrm{S})=150 \mathrm{~ms}, 65 \mathrm{~dB} \mathrm{SPL} \\
\mathrm{DIT}=0 \mathrm{~ms} \text {, puis } 1 \mathrm{~ms}\end{array}$ \\
\hline 10) SLT $(0-5-200) \mathrm{M}$ & $8 \mathrm{kHz}, 3 \mathrm{~ms}$ & $\Delta \mathrm{F}=100-15000 \mathrm{~Hz}, 250 \mathrm{~ms}, 60 \mathrm{~dB} \mathrm{SPL}$ \\
\hline 11) COLM & & $\Delta \mathrm{F}=100-15000 \mathrm{~Hz}, 350 \mathrm{~ms}, 65 \mathrm{~dB} \mathrm{SPL}, \tau\left(\mathrm{B} / \mathrm{B}^{\prime}\right)=10 \mathrm{~ms}$ \\
\hline 12) DLM & & $\Delta \mathrm{F}=100-15000 \mathrm{~Hz}, 300 \mathrm{~ms}$, Réf $=65 \mathrm{~dB}$ SPL \\
\hline 13) CTM & Glide $2-4 \mathrm{kHz}, 200 \mathrm{~ms}, 10 \mathrm{~dB} \mathrm{SL}$ & Glide $1-2 \mathrm{kHz}, 100 \mathrm{~ms}, \mathrm{R} / \mathrm{F} 15 / 5 \mathrm{~ms}$ \\
\hline 14) THM & & $\Delta \mathrm{F}=100-15000 \mathrm{~Hz}, 10 / 500 / 10 / 22 / 10 \mathrm{~ms}, 75 \mathrm{~dB} \mathrm{SPL}$ \\
\hline
\end{tabular}

\section{RÉSULTATS.}

Les unités employées pour exprimer les variables sont de deux types. Pour les seuils de détection (tests 7 à 14), les mesures ont été faites en décibels. Par contre pour les mesures de discrimination (tests 1 à 6) nous avons utilisé l'unité physique correspondante (durée en millisecondes et fréquence en hertz). Le seuil de discrimination de niveaux fait exception à la règle puisque la tradition en psychoacoustique veut qu'il soit exprimé en décibels. Cette différence entre unités de grandeur physique (ms, $\mathrm{Hz}$ ) et unités transformées $(\mathrm{dB})$ nous a conduits à faire une distinction dans la présentation des résultats.

\subsection{Présentation des résultats}

Les seuils différentiels suivent la loi de Weber, qui dit que pour une stimulation physique d'intensité $\mathrm{F}$, le seuil différentiel DF est proportionnel à $\mathrm{F}$. Il est donc naturel que les résultats des mesures correspondantes se répartissent statistiquement selon une distribution géométrique. Au contraire, il est normal que les seuils de détection suivent une distribution arithmétique. Cela s'observe très bien sur les histogrammes de seuils moyens lorsque l'on représente sur un même graphique à échelle linéaire la série des données mesurées et une série théorique "normale" ayant la même moyenne et le même écart type. Si la série de données est arithmétique, les deux histogrammes se superposent. Si au contraire la série est géométrique, le décalage entre eux sera net, puisque la moyenne géométrique et la moyenne arithmétique ne seront pas égales en général. C'est ce qui apparaît sur les deux figures qui suivent.

La Fig. 1 concerne les résultats de discrimination de durée; la Fig. 2 représente la détection d'un son pur dans un bruit (test $\mathrm{n}^{\circ} 8$ ) et la discrimination fréquentielle au voisinage de $1 \mathrm{kHz}$. Manifestement les résultats de discrimination de durée ne s'accomodent pas bien d'une représentation par une série normale, si l'on considère la variable mesurée en ms (Fig. 1, gauche). La série mesurée (en noir) et la série théorique (en gris) sont décalées l'une par rapport à l'autre. Par contre les logarithmes des résultats sont proches d'une distribution normale (Fig. 1, droite). D'autre part (Fig. 2) les seuils de détection exprimés en dB (échelle linéaire) ont d'entrée une répartition relativement symétrique et proche d'une loi normale, alors que pour les seuils de discrimination fréquentielle c'est sur une échelle logarithmique que leur distribution est normale. On notera au passage la dispersion considérable des seuils de discrimination fréquentielle. 

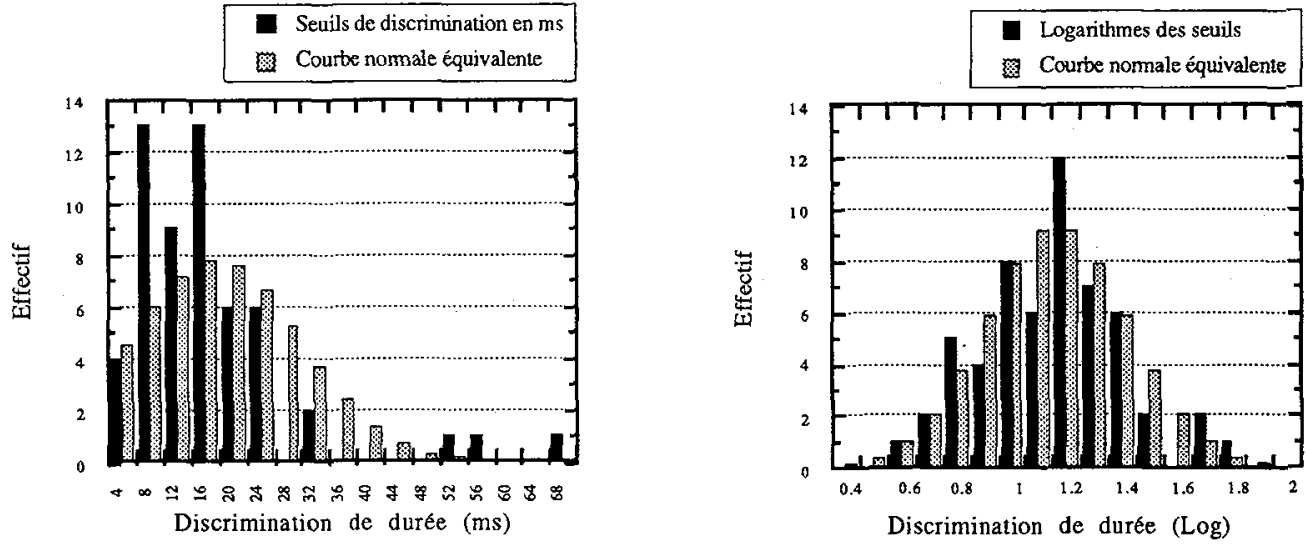

Figure 1. Graphe de gauche : histogramme des seuils de discrimination de durée. Les ordonnées correspondent aux effectifs réels de chaque classe (bandes noires) et aux effectifs théoriques d'une série normale équivalente, c'est-à-dire de même moyenne arithmétique et de même écart type (bandes grises). Graphe de droite : histogramme des logarithmes des seuils de discrimination de durée et distribution normale équivalente.
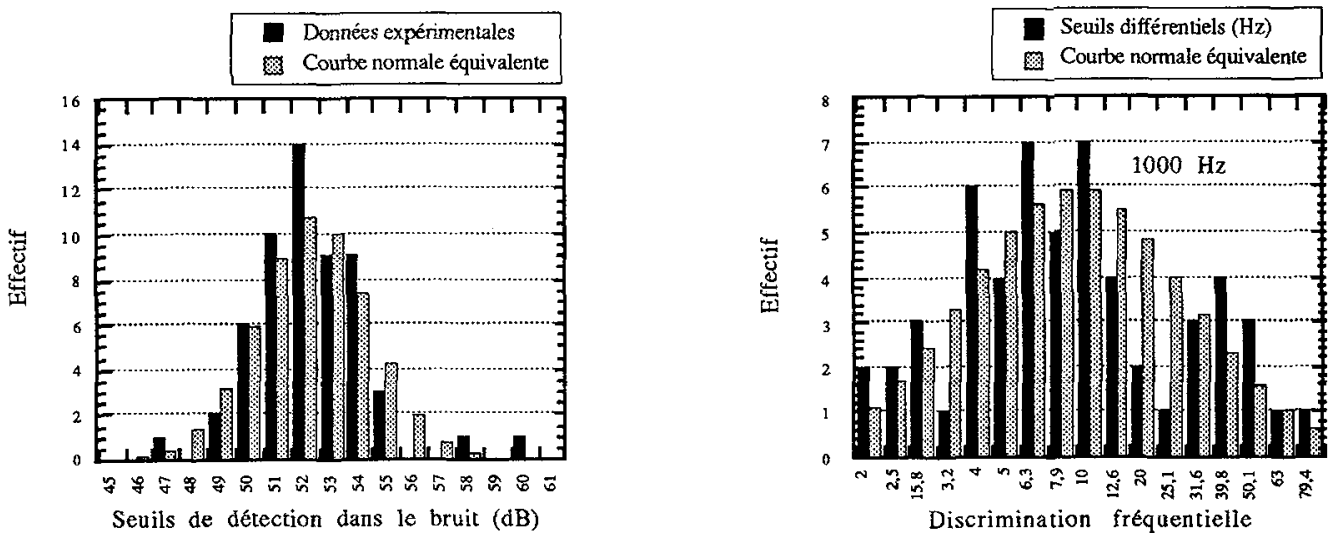

Figure 2. Graphe de gauche : histogramme des seuils de détection (test $\mathrm{n}^{\circ} 8$ ) et histogramme d'une distribution normale de même moyenne et de même écart type. Graphe de droite : histogramme des seuils de discrimination fréquentielle à $1000 \mathrm{~Hz}$, et courbe normale correspondante.

En conséquence, pour la suite de l'analyse statistique nous avons décidé d'utiliser des variables centrées réduites (ou $Z$-scores, de moyenne nulle et de variance unité) qui seront déterminées, suivant le cas, à partir des logarithmes des seuils (variables des tests 1 à 7), ou à partir des seuils eux-mêmes (autres variables).

\subsection{Seuils moyens et dispersion}

Une autre conséquence de la précédente remarque est que les séries à caractère géométrique doivent logiquement être représentées par leurs moyennes géométriques; et respectivement pour les séries arithmétiques. Il faut alors distinguer les expressions des écarts types : dans le cas des séries arithmétiques les écarts types $(\sigma)$ seront des constantes additives $\left(X_{i}=X_{g}+\sigma\right)$, et dans le cas des séries géométriques ce seront des constantes multiplicatives $\left(X_{i}=X_{g} * k\right)$. Pour rappeler cette distinction, nous avons désigné les écarts types géométriques par le symbole ${ }^{*}$ dans le tableau II ci-dessous, qui donne un sommaire des résultats. 
Tableau II. Valeurs moyennes des seuils et paramètres de dispersion pour les différents tests.

\begin{tabular}{|l|c|c|c|c|}
\hline Variables & Moyennes & Ecarts types & Valeurs minimales & Valeurs maximales \\
\hline 1) DTM & $14,1 \mathrm{~ms}$ & $* 1,81$ & 3,7 & 69 \\
\hline 2) DGM & $25,5 \mathrm{~ms}$ & $* 1,83$ & 6,9 & 143 \\
\hline 3) RYM & $9,9 \mathrm{~ms}$ & $* 1,64$ & 4 & 31,5 \\
\hline 4) FUM & $562 \mathrm{~Hz}$ & $* 2,37$ & 56 & - \\
\hline 5) DF1KM & $11 \mathrm{~Hz}$ & $* 2,52$ & 2,2 & 77 \\
\hline 6) DF4KM & $42 \mathrm{~Hz}$ & $* 3,14$ & 6,3 & 990 \\
\hline 7) TSM & $27 \mathrm{~ms}$ & $* 1,6$ & 8,1 & 63 \\
\hline 8) TTM & $56 \mathrm{~dB}$ & 24 & 7 & 86 \\
\hline 9) BR1KM & $52 \mathrm{~dB}$ & 2,04 & 47 & 60 \\
\hline 10) BR18M & $19,6 \mathrm{~dB}$ & 3,9 & 9,5 & 28,8 \\
\hline 11) MLDOM & $55,6 \mathrm{~dB}$ & 2,36 & 51 & 61 \\
\hline 12) MLD1M & $39,6 \mathrm{~dB}$ & 4,8 & 16 & 57 \\
\hline 13) SLT200M & $67,4 \mathrm{~dB}$ & 3,5 & 61,7 & 76,7 \\
\hline 14) SLT5M & $52,5 \mathrm{~dB}$ & 11,7 & 31 & 74 \\
\hline 15) SLTOM & $83,4 \mathrm{~dB}$ & 7,8 & 65,7 & -- \\
\hline 16) COLM & $-12,5 \mathrm{~dB}$ & 4,5 & -19 & 5 \\
\hline 17) DLM & $0,98 \mathrm{~dB}$ & 0,56 & 0,22 & 2,9 \\
\hline 18) CTM & $49 \mathrm{~dB}$ & 17 & 10 & 85 \\
\hline 19) THM & $58 \mathrm{~dB}$ & 9,8 & 40 & 80 \\
\hline
\end{tabular}

La dispersion des données est habituellement représentée par les écarts types des mesures. Mais on tire également de précieux renseignements des valeurs extrêmes des séries. Nous avons donc porté ces indications dans les deux colonnes de droite du tableau II. Pour les variables $n^{\circ} 4$ (fusion interaurale) et $n^{\circ} 15$ (sélectivité temporelle) les valeurs supérieures manquent. Cela provient de ce que certains sujets (respectivernent quatre pour la fusion et deux pour la sélectivité) ne sont pas parvenus à faire le test, dans les limites que nous avions fixées pour les variables correspondantes.

Enfin, dans certains cas il est instructif de pouvoir examiner le détail de la répartition des seuils. Nous présentons donc ci-dessous les histogrammes des données de deux des tests pour lesquels la dispersion s'est révélée la plus forte (masquage antérieur, test $n^{\circ} 10$ et masquage informationnel, test $n^{\circ} 7$ ). C'est aussi en raison de cette forte dispersion que nous avons choisi de présenter les résultats de discrimination fréquentielle à la Fig. 2 .

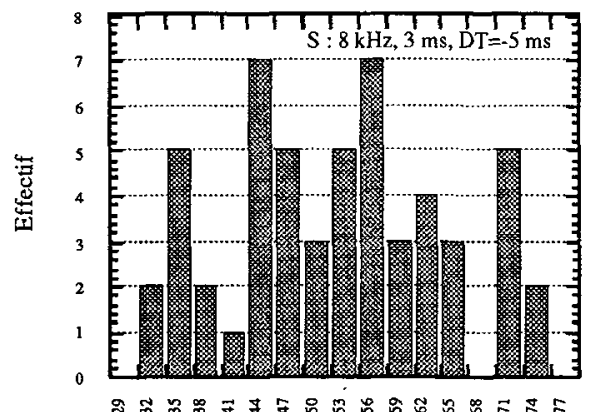

Seuil de détection ( $\mathrm{dB}$ ) d'un clic de $8 \mathrm{kHz}$ dans le bruit

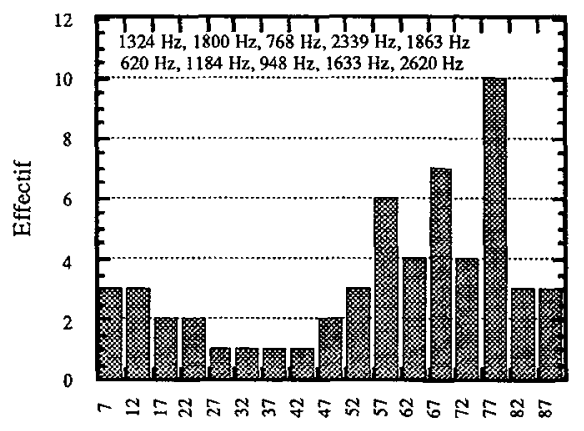

Seuils de masquage informationnel (dB)

Figure 3. Histogrammes des résultats de mesures de sélectivité temporelle par masquage rétroactif à gauche (test $n^{\circ} 10$, décalage des attaques entre signal et masque : $5 \mathrm{~ms}$ ), et des mesures de masquage informationnel à droite. 


\section{ANALYSE DISCRIMINANTE.}

Le premier objectif de ce type d'étude est d'établir les limites normales des facultés auditives testées. Mais la question qui se pose immédiatement est de savoir comment se distribue la population normale à l'intérieur de ces limites, et en particulier de savoir s'il existe des partitions possibles en groupes cohérents, et éventuellement indépendants, du point de vue auditif. A partir d'un grand nombre de données, cette recherche se fait habituellement par la technique dite des "clusters".

\subsection{Exemple de classification}

Les résultats de la première étude [1] avaient déjà montré que les capacités auditives semblent réparties entre les individus de manière presque "continue". Autrement dit, les résultats des auditeurs aux différents tests s'échelonnent continûment entre les limites des séries obtenues. Cette répartition continue se retrouve si l'on représente chaque sujet par un vecteur à n composantes (n étant égal au nombre de variables testées) et que l'on considère l'ensemble des $\mathrm{N}$ vecteurs ainsi obtenus ( $\mathrm{N}=$ nombre de sujets testés) ou le nuage de points correspondant : il ne se forme pas d'amas nettement différenciés, et l'on obtient plutôt un nuage de points. C'est la conclusion que l'on peut tirer des résultats de l'analyse discriminante que nous avons faite. Etant donné les valeurs manquantes à certains tests; il a fallu procéder à cette analyse en excluant cinq sujets; donc $\mathrm{N}=51$ ici, et $\mathrm{n}=19$.

Pour effectuer l'analyse discriminante, nous avons utilisé une méthode de regroupement qui consiste à réunir en un seul sous-groupe deux sujets dont la distance dans l'espace à $\mathrm{n}$ dimensions mentionné plus haut est la plus petite de toutes les distances entre sujets. Puis on adjoint au sous-groupe ainsi formé le sujet qui en est le plus proche, ou bien on forme un nouveau sous-groupe à deux individus, et ainsi de suite.

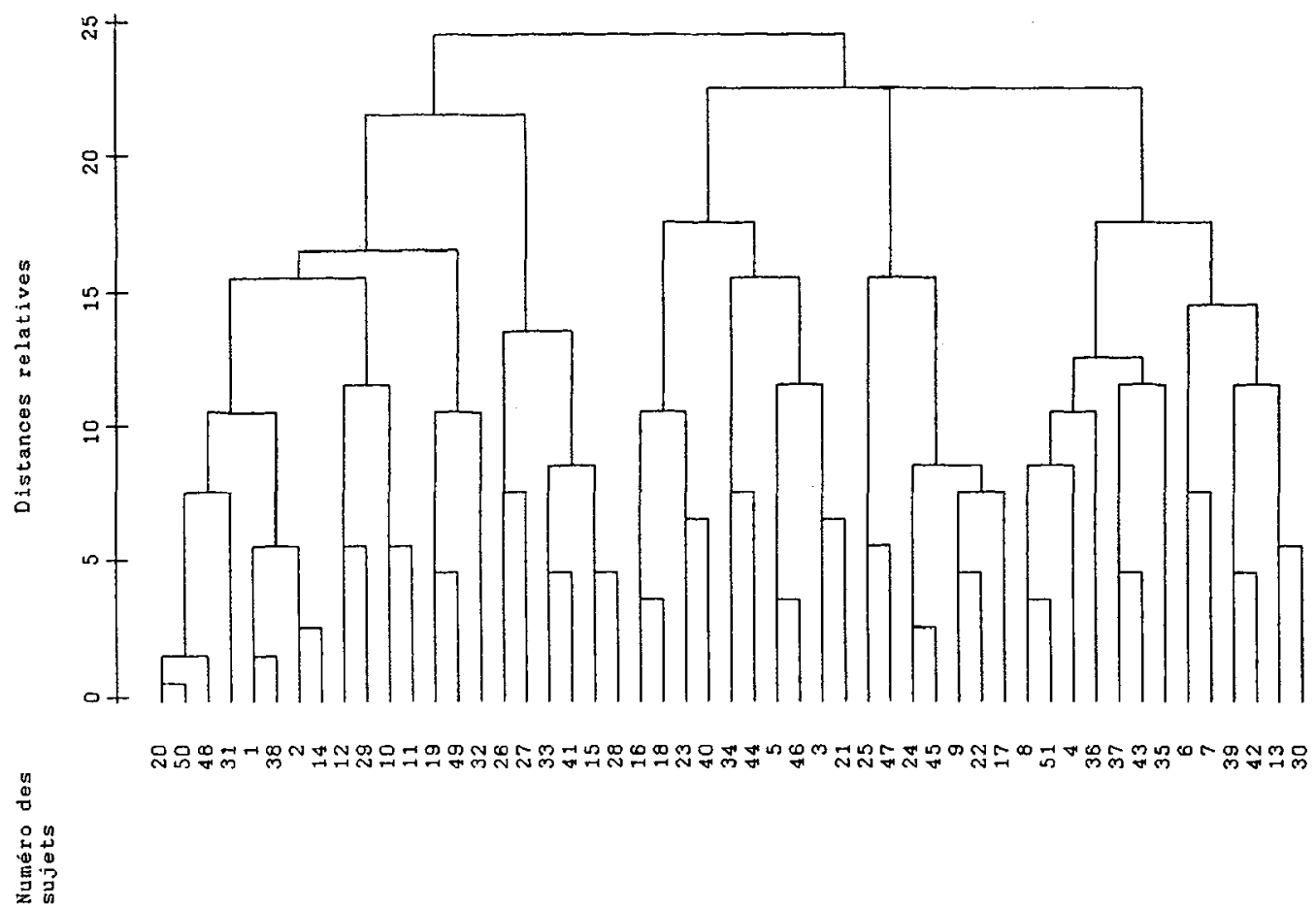

Figure 4. Arbre de classification de 51 des sujets testés, d'après leurs distances relatives dans l'espace des 19 variables.

Le résultat d'une telle analyse peut être représenté sous la forme d'un arbre de classification comme celui de la Fig.4. L'abscisse de ce graphique porte les numéros des sujets, et l'ordonnée indique la distance qui les sépare (en valeur relative). La distance peut se définir de différentes manières; nous avons choisi une grandeur proportionnelle au cosinus de l'angle entre vecteurs (sujets). Il y a deux façons d'examiner cet 
arbre, suivant que l'on part du haut ou du bas du graphique. Nous commencerons par le bas, en observant les regroupements effectués. En partant de la gauche du graphique, on voit que ce sont les sujets $\mathrm{n}^{\circ} 20 \mathrm{et}^{\circ}$ 50 (à gauche du graphe) qui sont les plus proches. Leur distance correspond à l'ordonnée du segment de droite horizontal qui les relie. Ils forment donc le premier regroupement. Mais tout de suite après on trouve le sujet $n^{\circ} 48$, qui n'en est pas très éloigné non plus. Plus précisément cela signifie que les résultats de ces trois sujets aux tests ont été les plus voisins. Ces trois sujets ont constitué le premier "cluster". Ensuite on peut voir que ce sont les sujets 1 et 38 qui sont les plus proches, puis la paire 2-14, etc.

\subsection{Le regroupement par catégories}

Une autre façon d'analyser les résultats consiste à partir du haut du graphique. Les groupes que l'on peut former dans notre échantillon d'auditeurs sont, en quelque sorte, les "branches" qui tombent de l'arbre de classification lorsque l'on coupe les traits verticaux à des niveaux d'agrégation de plus en plus bas. Par exemple, si l'on coupe l'arbre à la hauteur de l'ordonnée 24 , on sépare les 51 sujets en deux ensembles. Le premier va du sujet $n^{\circ} 20$ au sujet $n^{\circ} 28$ (voir les abscisses) et contient donc 21 personnes. Le second ensemble est le complémentaire du premier; il va du sujet $n^{\circ} 16$ au sujet $n^{\circ} 30$, soit au total 30 personnes.

D'un certain point de vue, ces deux ensembles regroupent les auditeurs qui sont les plus "semblables". Mais on peut affiner la classification, et couper l'arbre à une hauteur inférieure; à une ordonnée de 20 par exemple. On obtient alors cinq groupes. Ils vont respectivement des sujets $\mathrm{n}^{\circ} 20$ à 32 (15 sujets), 26 à 28 (6 sujets), 16 à 21 (10 sujets), 25 à 17 (7 sujets), et 8 à 30 (13 sujets).

Il est alors possible d'apprécier les caractéristiques des différents groupes, en calculant par exemple les moyennes globales de chaque groupe à chacun des tests effectués. C'est ce qui apparaît sur la figure cidessous, où les abscisses représentent les numéros des variables telles qu'elles sont définies dans le tableau II. Ce sont ces lignes brisées que nous appelons profils.

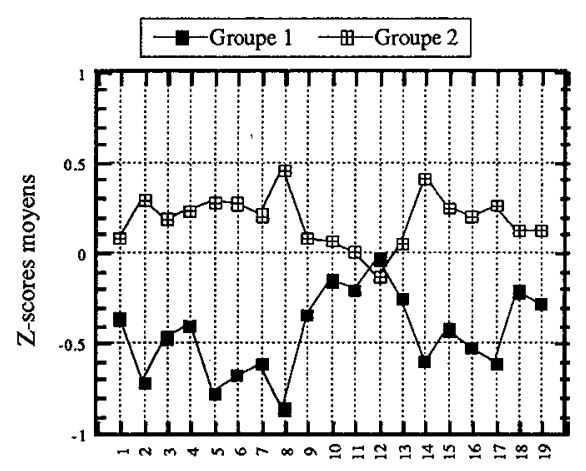

Numéro de la variable

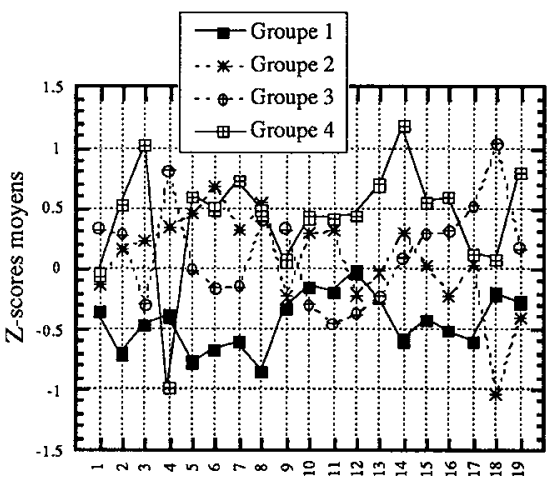

Numéro de la variable

Figure 5. Profils psychoacoustiques moyens des groupes résultant de l'analyse discriminante : répartition en deux groupes à gauche, et en quatre groupes à droite.

Sur la figure précédente, les ordonnées sont exprimées en unités d'écart type des variables réduites. On sait que, par définition, la moyenne des données réduites ( $\mathrm{Z}$-scores) de l'ensemble des sujets pour chaque test est égale à 0 , et l'écart type égal à 1 . Donc c'est le fractionnement en deux groupes qui donne des moyennes non nulles à chacun des groupes; par exemple pour la huitième variable (TTM) les moyennes respectives sont d'environ 0,5 pour le groupe 2 et $-0,9$ pour le groupe 1 .

Ce graphique fait immédiatement apparaître les tests qui sont probablement classifiants et ceux qui ne le sont pas. Par exemple, les variables de 10 à 13 donnent des scores très voisins pour les deux groupes. Ce sont des tests de détection de son pur dans le bruit, et comme on l'a déjà vu ces tests donnent rarement des résultats à forte variabilité. Par contre les variables 5 à 8 (tests liés à la fréquence) séparent très nettement les deux groupes. 
Si l'on veut approfondir l'analyse, on peut calculer les moyennes associées à une partition en quatre groupes (Fig. 5, graphe de droite). Cette nouvelle présentation ne change pas les résultats du groupe 1. En effet, le sectionnement de l'arbre de classification à ce niveau d'agrégation (environ 22 ou 23 sur la figure 4) n'affecte que la première branche de droite, qui se voit décomposée en trois sous-ensembles. A part pour la variable $\mathrm{n}^{\circ} \mathbf{4}$ (fusion), le groupe 4 forme alors une sorte de limite supérieure des scores, le groupe 1 formant la limite inférieure, qui en fait correspond aux seuils les plus bas donc aux performances les meilleures.

Dans la mesure où nous avons choisi une représentation graphique des scores relatifs, les courbes moyennes ne s'éloigneront pas beaucoup de l'intervalle d'ordonnées allant de -1 à +1 . En effet, ces scores étant justement calculés pour avoir un écart type de 1, les deux tiers environ de la population se répartiront normalement entre -1 à +1 . Par contre, si l'on veut entrer dans le détail des performances individuelles, on trouvera obligatoirement des sujets qui s'écarteront nettement de ces limites.

\subsection{Comparaison de profils particuliers}

Pour cette comparaison des profils individuels, nous avons choisi un sujet du groupe 1 et un sujet du groupe 4. On appréciera sur la Fig. 6 l'étendue des écarts entre scores individuels (de -2 à +2 environ), et aussi la variabilité, pour une même personne, des performances auditives.

Les profils peuvent également être observés entre classes particulières, telles que hommes et femmes par exemple, ou musiciens et non musiciens. Parmi les sujets que nous avons testés, rares étaient les vrais musiciens, ayant une formation et une pratique solides de la musique. Nous avons donc adopté la règle suivante pour le partage de notre échantillon : étaient considérés comme musiciens tous ceux qui pratiquaient, même occasionnellement, un instrument de musique ou le chant. Les autres sujets constituaient le sous-ensemble des non-musiciens.
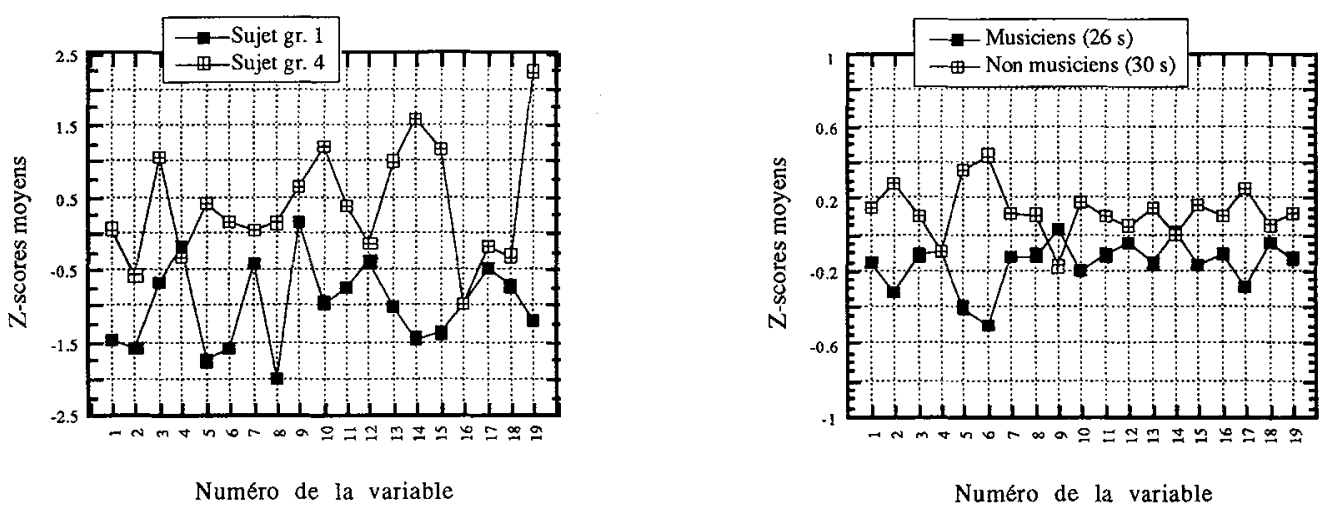

Figure 6. Profils psychoacoustiques individuels de deux sujets (l'un du groupe 1, l'autre du groupe 4) à gauche; comparaison des profils moyens des sujets musiciens et des non musiciens à droite.

Il est certain qu'une telle séparation n'est pas exigeante pour la qualité de musicien. On peut donc s'attendre à une faible différence de scores entre les deux sous-ensembles ainsi constitués. Effectivement le test de Student confirme qu'il n'y a pas de différence significative entre les deux, excepté pour les tests de discrimination fréquentielle. Le graphique de droite Fig. 6 traduit aussi la même idée, quoique la différence, même minime, soit toujours dans le même sens : celui d'une légère supériorité des sujets musiciens.

\section{CONCLUSION.}

Le premier apport important de cette étude sur les différences auditives individuelles réside dans la cartographie qu'elle fournit des performances normales à une série assez diversifiée de tests. Sans avoir de prétentions normatives, les données recueillies forment tout de même une base de référence pour la dispersion normale des caractéristiques auditives testées, dans les conditions expérimentales indiquées. 
Un second point concerne l'exploitation que l'on peut faire de la variabilité de cette dispersion suivant les tests considérés. Comme on le savait déjà, les tests classiques de détection dans le bruit produisent des résultats peu différents (relativement) entre les individus; la dynamique est de l'ordre de 10 $\mathrm{dB}$, et les écarts types ne sont que de 2 à $3 \mathrm{~dB}$. Par contre on a montré ici combien certains tests, notamment les $n^{\circ} 5,7,10$ et 11 , pouvaient être dispersifs, et donc sélectifs pour l'établissement d'un bilan audiométrique. A ce stade déjà, une batterie minimale constituée de ces tests fortement dispersifs pourrait donc trouver des applications professionnelles très utiles.

Enfin, de par leur contribution prépondérante à la variance globale de nos données, révélées par l'analyse en composantes principales que nous avons faite, les variables correspondant à ces tests peuvent être retenues, dans une première étape, pour constituer les vecteurs représentatifs dont il a été question dans l'introduction. Le cadre du profil psychoacoustique pourrait alors être limité à ces quelques variables à forte dispersion.

Il faut toutefois poursuivre l'analyse, notamment pour évaluer le degré d'indépendance de ces variables et donc des vecteurs qu'elles pourraient représenter. S'ils sont effectivement retenus pour constituer la base recherchée, il faudra de toute façon leur adjoindre quelques autres facteurs auditifs fondamentaux, qui restent à définir, entre autres des facteurs plus centraux ou des facteurs à caractère perceptif.

\section{BIBLIOGRAPHIE.}

[1] Canévet G. Les profils psychoacoustiques. Dans : "Genèse et perception des sons", publications du L.M.A., $\mathrm{n}^{\circ} 128$, octobre 1991 .

[2] Carton P. Diagnostic et conduite des tempéraments. Ed. N. Maloine, Paris, 1926.

[3] Fastl H. Temporal Masking Effects : I. Broad Band Noise Masker. Acustica, 1976, 35, 288-302.

[4] Johnson D.M., Watson C.S., Jensen J.K. Individual differences in auditory capabilities. I. J. Acoust. Soc. Amer. 1987, 81, 2, 427-438.

[5] Kuder G.F. Kuder preference record, occupational, form D. Science Research Associate, Chicago, 1956.

[6] Santon F. Coloration d'un bruit blanc et de bandes de bruit apportée par un écho de même direction frontale. J. Acoustique 1991, 4, 237-252.

[7] Trenque P., König E. The Chirp Train Streaming Test. A Clinical Approach to Auditory Selectivity and Listening. Audiology, 1988, 27, 65-88.

[8] Watson C.S. Uncertainty, informational masking and the capacity of immediate auditory memory. In W.A. Yost et C.S. Watson (Eds) Auditory Processing of Complex Sounds, Erlbaum Associates, 1987, Hillsdale, New Jersey.

(Cette étude a été réalisée dans le cadre d'une convention de recherche avec la DCN de Toulon et le CERDSM, Le Brusc) 